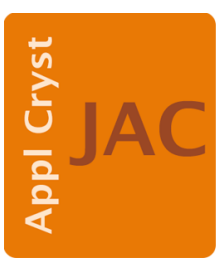

JOURNAL OF

APPLIED

CRYSTALLOGRAPHY

Volume 51 (2018)

Supporting information for article:

Evidence of new twinning modes in magnesium questioning the shear paradigm

Cyril Cayron and Roland Logé 


\section{Evidence of new twinning modes in magnesium questioning the shear paradigm}

Authors

\section{Cyril Cayron $^{\text {a* }}$ and Roland Logéa}

aMX LMTM, Ecole Polytechnique Fédérale de Lausanne (EPFL), Rue de la Maladière, 71b, Neuchâtel, 2000, Switzerland

Correspondence email: cyril.cayron@epfl.ch

\section{Supporting Figures}

a

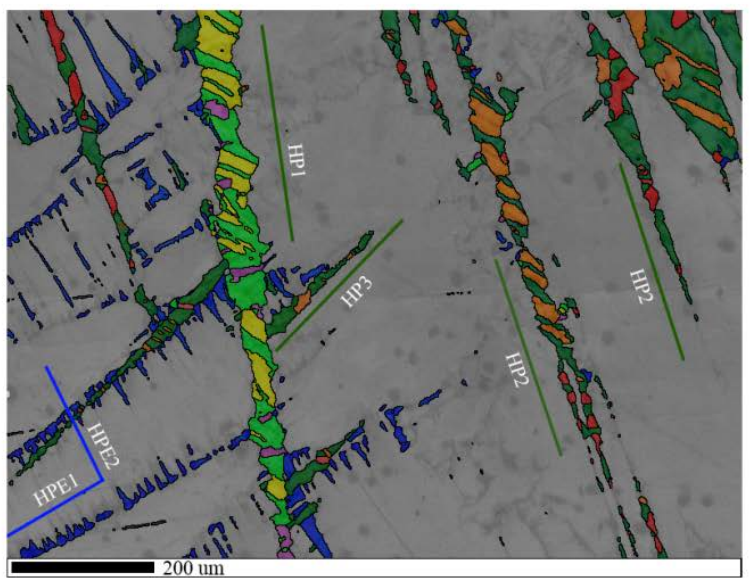

$\mathrm{b}$

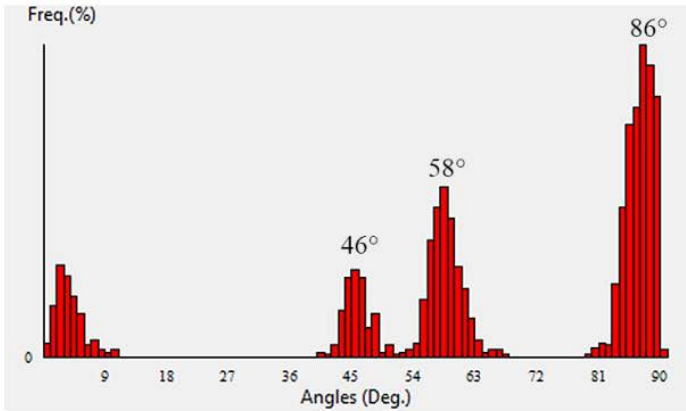

$\mathrm{c}$
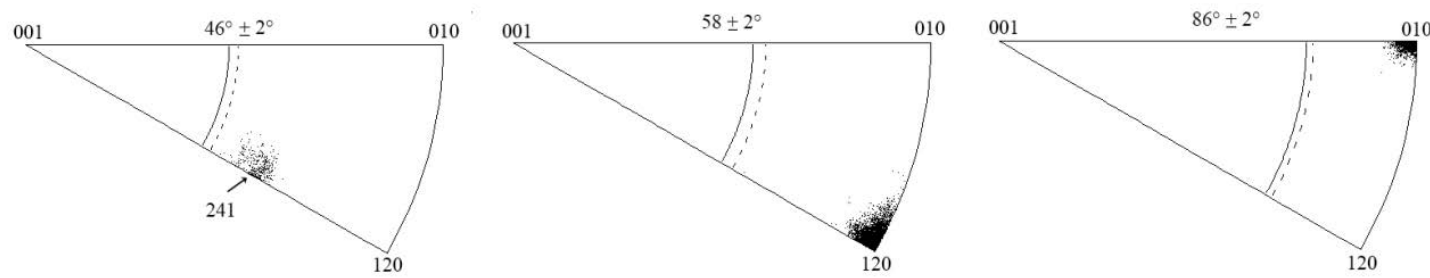

Figure S1 EBSD map of the magnesium single crystal cut on section B. (a) Map with colors chosen according to those used for the different twin types in Figure1a. (b) Disorientation histogram, with in (c) the rotation axes corresponding to the three peaks of the histogram plotted in the fundamental sector of the hexagonal lattice. 

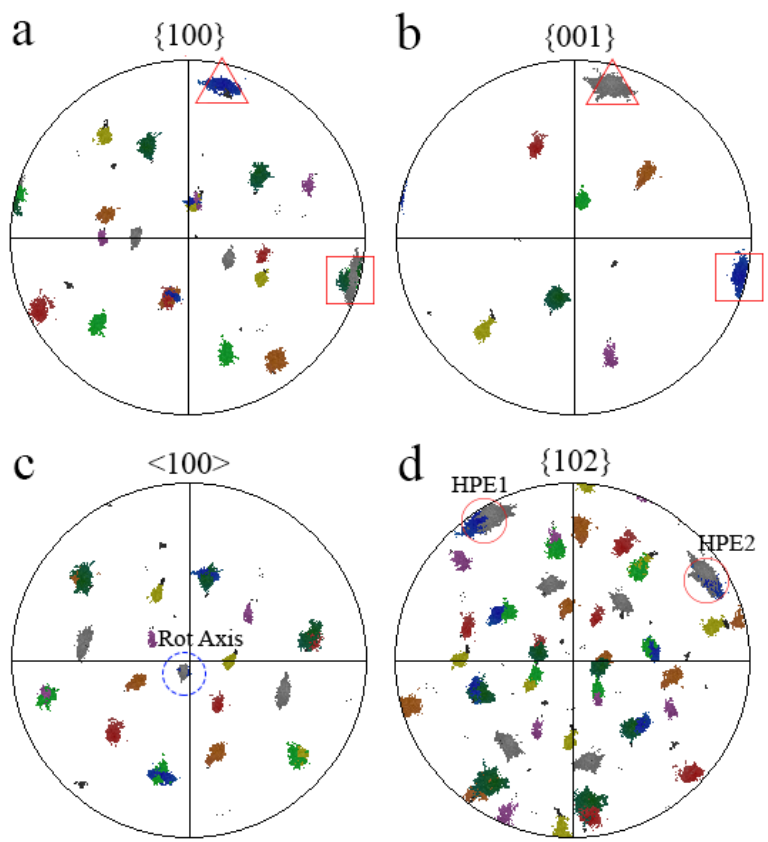

Figure S2 Pole figures of the EBSD map shown in Figure S1 indicating some important crystallographic planes and directions related to the $\left(86^{\circ}, \mathbf{a}\right)$ extension twin. The planes and directions marked by the rectangles, triangles and circles are interpreted exactly as in Figure2.
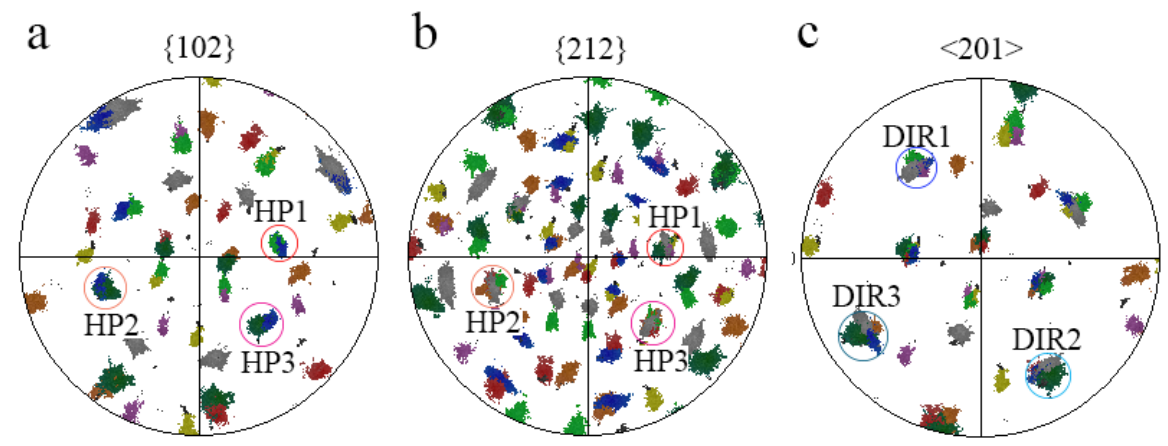

Figure S3 Pole figures of the EBSD map shown in Figure S1 confirming the unconventional character of the $\sim\left(58^{\circ}, \mathbf{a}+2 \mathbf{b}\right)$ green twins already shown in Figure3. Two variants of $\sim\left(58^{\circ}, \mathbf{a}+2 \mathbf{b}\right)$ twins are visible in this map (light and dark green): the dark green twins exhibit two different habit planes, and the light green twin only has one habit plane. These three habit planes, noted HP1, HP2 and HP3, are perfectly indexed as $\{212\}_{p} / /\{012\}_{g r}$ planes. It was also checked that all these twins share a common $\langle 201\rangle$ direction, noted DIR1, DIR2 and DIR3, that belongs to the $\{212\}$ plane HP1, HP2 and HP3, respectively. 
a

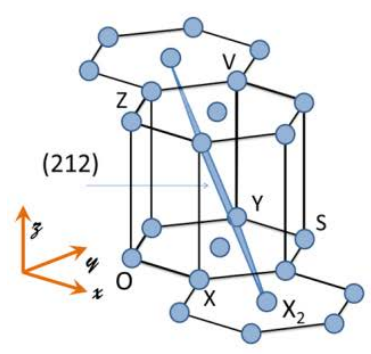

b

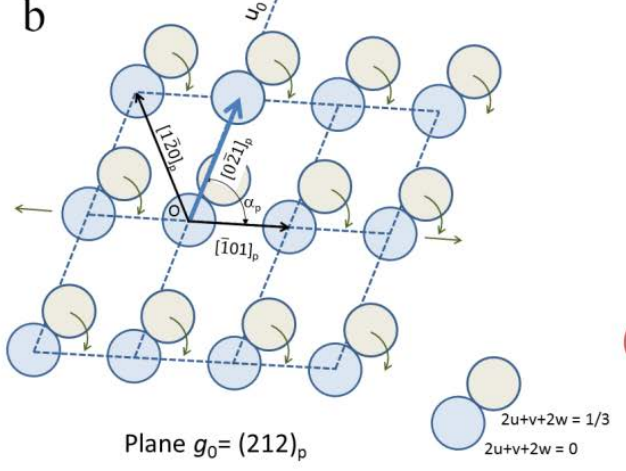

$\mathrm{c}$

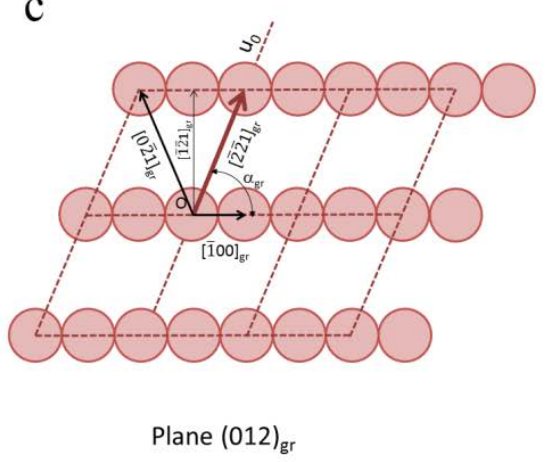

Figure S4 Atomic model of the $(212)_{\mathrm{p}} \rightarrow(012)_{\mathrm{gr}}$ transformation. (a) Plane (212) $)_{\mathrm{p}}$ of the parent crystal drawn in the reference frame. (b) The same plane viewed edge-on; it is constituted of two parallel layers of atoms, one with atoms of coordinates $[u, v, w]$ positioned at the level $l=2 u+v+2 w=$ 0 (in blue), and the other one with atoms at the level $l=2 u+v+2 w=1 / 3$ (in light grey). The displacements of the atoms of the layer $l=1 / 3$ down to the lower layer $l=0$ are shown by the green curved arrows. (c) Plane (012) gr $_{\text {of }}$ the green twin constituted of only one layer $\mathrm{l}=\mathrm{v}+2 \mathrm{w}=0$ (in red), obtained after the atomic displacements and lattice distortion. 


\section{Supporting Calculations}

\section{S1. Reminder on some important crystallographic matrices of phase transformation}

\section{S1.1. Definition of the distortion, orientation and correspondence matrices}

Deformation twinning is a lattice transformation under stress or strain from a parent crystal (p) to the twinned crystal ( $\mathrm{t}$ ); this distortion restores the lattice in a new orientation. Mathematically, it can be defined by a distortion matrix $\mathbf{D}^{p \rightarrow t}$. Any direction $\boldsymbol{u}$ is transformed after distortion into a new direction $\boldsymbol{u}^{\prime}=\mathbf{D}^{p \rightarrow t} \boldsymbol{u}$. A plane $\boldsymbol{g}$, considered as a vector of the reciprocal space, is transformed after distortion into a new plane $\boldsymbol{g}^{\prime}=\left(\mathbf{D}^{p \rightarrow t}\right)^{*} \boldsymbol{g}$ with $\left(\mathbf{D}^{p \rightarrow t}\right)^{*}=\left(\mathbf{D}^{p \rightarrow t}\right)^{-\mathrm{T}}$ where the symbol $-\mathrm{T}$ means the inverse of the transpose.

It is often necessary for the calculation to switch from the crystallographic basis to an orthonormal basis linked to this basis. In the case of an hexagonal phase, we call $\mathbf{B}_{\text {hex }}=(\boldsymbol{a}, \boldsymbol{b}, \boldsymbol{c})$ the usual hexagonal basis, and $\mathbf{B}_{\text {ortho }}=(\boldsymbol{x}, \boldsymbol{y}, \boldsymbol{z})$ the orthonormal basis linked to $\mathbf{B}_{\text {hex }}$ by the coordinate transformation matrix $\mathbf{H}_{\text {hex }}$ :

$$
\mathbf{H}_{\text {hex }}=\left[\mathbf{B}_{\text {ortho }} \rightarrow \mathbf{B}_{\text {hex }}\right]=\left(\begin{array}{ccc}
1 & -1 / 2 & 0 \\
0 & \sqrt{3} / 2 & 0 \\
0 & 0 & \gamma
\end{array}\right)
$$

where $\gamma$ is the c/a packing ratio of the hexagonal phase. The matrix $\mathbf{H}_{\text {hex }}$ is commonly called structure tensor in crystallography. It can be used to express the directions into the orthonormal basis $\mathbf{B}_{\text {ortho }}$. For planes, it is $\mathbf{H}_{\text {hex }}^{*}$ that should be used. We note O, the "zero" position that will be left invariant by the distortion and we note $\mathrm{X}, \mathrm{Y}$ and $\mathrm{Z}$ the atomic positions defined by the vectors $\mathbf{O X}=\boldsymbol{a}$ $=[100]_{\text {hex }}, \mathbf{O Y}=\boldsymbol{a}+2 \boldsymbol{b}=[120]_{\text {hex }}$ and $\mathbf{O Z}=\boldsymbol{c}=[001]_{\text {hex }}$. It can be checked with the matrix $\mathbf{H}_{\text {hex }}$ that $\mathbf{O X}=[100]_{\text {ortho, }}, \mathbf{O Y}=\left[\begin{array}{lll}0 & \sqrt{3} & 0\end{array}\right]_{\text {ortho }}$ and $\mathbf{O Z}=\left[\begin{array}{lll}0 & 0 & \gamma\end{array}\right]_{\text {ortho. }}$.

The vectors of the initial parent basis are transformed by the distortion into new vectors: $\mathbf{a}_{p} \rightarrow \mathbf{a}^{\prime}{ }_{p}$, $\mathbf{b}_{p} \rightarrow \mathbf{b}^{\prime}{ }_{p}$ and $\mathbf{c}_{p} \rightarrow \mathbf{c}^{\prime}{ }_{p}$. The distortion matrix expressed in the hexagonal basis $\mathbf{D}_{h e x}^{p \rightarrow t}$ is the matrix formed by the images $\mathbf{a}^{\prime}{ }_{p}, \mathbf{b}^{\prime}{ }_{p}$ and $\mathbf{c}^{\prime}{ }_{p}$ expressed in $\mathbf{B}_{\text {hex }}$, i.e. $\mathbf{D}_{\text {hex }}^{p \rightarrow t}=\left[\mathbf{B}_{\text {hex }}^{p} \rightarrow \mathbf{B}_{\text {hex }}^{\prime p}\right]=\mathbf{B}_{\text {hex }}^{\prime p}$ with $\mathbf{B}_{h e x}^{p}=\left(\mathbf{a}_{p}, \mathbf{b}_{p}, \mathbf{c}_{p}\right)$ and $\mathbf{B}_{h e x}^{\prime p}=\left(\mathbf{a}^{\prime}{ }_{p}, \mathbf{b}^{\prime}{ }_{p}, \mathbf{c}^{\prime}{ }_{p}\right)$. In simple words, the distortion matrix is expressed by writing in column the coordinates of $\mathbf{a}_{p}^{\prime}, \mathbf{b}_{p}^{\prime}$ and $\mathbf{c}^{\prime}{ }_{p}$ in the basis $\mathbf{B}_{h e x}^{p}$. The crystallographic studies on displacive phase transformations and mechanical twinning often consist in finding the distortion matrices close to the identity matrix in order to minimize the atomic displacements.

If the distortion matrix is known in the basis $\mathbf{B}_{\text {ortho }}$, and noted $\mathbf{D}_{\text {ortho }}^{p \rightarrow t}$, a formula of coordinate transformation can be used to express it in the basis $\mathbf{B}_{\text {hex }}$; it is: 


$$
\mathbf{D}_{\text {hex }}^{p \rightarrow t}=\mathbf{H}_{\text {hex }}^{-1} \mathbf{D}_{\text {ortho }}^{p \rightarrow t} \mathbf{H}_{\text {hex }}
$$

with $\mathbf{H}_{\text {hex }}$ given by equation S1. Inversely, if the distortion matrix is found in $\mathbf{B}_{\text {hex }}$ and it can be written in $\mathbf{B}_{\text {ortho }}$ by the inverse formula:

$$
\mathbf{D}_{\text {ortho }}^{p \rightarrow t}=\mathbf{H}_{\text {hex }} \mathbf{D}_{\text {hex }}^{p \rightarrow t} \mathbf{H}_{\text {hex }}^{-1}
$$

The misorientation matrix is defined by the coordinate transformation matrix $\mathbf{T}_{h e x}^{p \rightarrow t}$. This matrix allows the change of the coordinates of a fixed vector between the parent and twin bases. It is given by the vectors forming the basis of the twin $\mathbf{B}_{\text {hex }}^{t}=\left(\mathbf{a}_{t}, \mathbf{b}_{t}, \mathbf{c}_{t}\right)$ expressed in the parent hexagonal basis, i.e. $\mathbf{T}_{\text {hex }}^{p \rightarrow t}=\left[\mathbf{B}_{\text {hex }}^{p} \rightarrow \mathbf{B}_{\text {hex }}^{t}\right]$. Its reverse is just $\mathbf{T}_{\text {hex }}^{t \rightarrow p}=\left[\mathbf{B}_{\text {hex }}^{t} \rightarrow \mathbf{B}_{\text {hex }}^{p}\right]$.

The orientation of the twinned crystal is defined by the matrix $\mathbf{T}_{h e x}^{p \rightarrow t}$, but other equivalent matrices could be chosen. The equivalent matrices are obtained by multiplying $\mathbf{T}_{h e x}^{p \rightarrow t}$ by the matrices $\boldsymbol{g}_{\boldsymbol{i}}$ of internal symmetries of the hexagonal phase, i.e. the matrices forming the point group of the hcp phase $\mathbb{G}^{h c p}$.

$$
\left\{\mathbf{T}_{\text {hex }}^{p \rightarrow t}\right\}=\left\{\mathbf{T}_{\text {hex }}^{p \rightarrow t} \boldsymbol{g}_{\boldsymbol{i}}, \boldsymbol{g}_{\boldsymbol{i}} \in \mathbb{G}^{h c p}\right\}
$$

The matrix $\mathbf{T}_{h e x}^{p \rightarrow t}$ is a coordinate transformation matrix between two hexagonal bases; it is thus a rotation matrix. The rotation angle of a matrix $\mathbf{T}_{h e x}^{p \rightarrow t}$ is given by its trace and the rotation axis is the eigenvector associated with the unit eigenvalue. However, one must keep in mind that $\mathbf{T}_{h e x}^{p \rightarrow t}$ is expressed in a non-orthonormal basis, which implies that some usual equations related to rotations do not hold. For example, the inverse of a rotation matrix equals its transposes only in orthonormal basis. Using $\mathbf{T}_{\text {ortho }}^{p \rightarrow t}=\mathbf{H}_{\text {hex }} \mathbf{T}_{\text {hex }}^{p \rightarrow t}\left(\mathbf{H}_{\text {hex }}\right)^{-1}$ in the calculations allow avoiding possible errors.

In the set of equivalent matrices $\left\{\mathbf{T}_{\text {hex }}^{p \rightarrow t}\right\}$, it is custom to choose the rotation with the lowest angle, called “disorientation”. This choice has practical applications, but it remains arbitrary.

The correspondence matrix $\mathbf{C}_{\text {hex }}^{t \rightarrow p}$ gives the distortion images expressed in the twin basis of the parent basis vectors, i.e. $\mathbf{a}_{p}^{\prime}, \mathbf{b}_{p}^{\prime}$ and $\mathbf{c}_{p}^{\prime}$. These images are obtained from the misorientation matrix and the distortion matrix: $\left(\mathbf{a}_{p}^{\prime}, \mathbf{b}_{p}^{\prime}, \mathbf{c}_{p}^{\prime}\right)_{/ \mathbf{B}_{\text {hex }}^{t}}=\mathbf{T}_{\text {hex }}^{t \rightarrow p}\left(\mathbf{a}_{p}^{\prime}, \mathbf{b}_{p}^{\prime}, \mathbf{c}_{p}^{\prime}\right)_{/ \mathbf{B}_{\text {hex }}^{p}}=\mathbf{T}_{\text {hex }}^{t \rightarrow p} \mathbf{B}_{\text {hex }}^{p}=\mathbf{T}_{\text {hex }}^{t \rightarrow p} \mathbf{D}_{\text {hex }}^{p \rightarrow t}$. The correspondence matrix is thus:

$$
\mathbf{C}_{\text {hex }}^{t \rightarrow p}=\mathbf{T}_{\text {hex }}^{t \rightarrow p} \mathbf{D}_{\text {hex }}^{p \rightarrow t}
$$

The correspondence matrix is used to calculate in the twin basis the coordinates of the image by the distortion of a vector written in the parent basis, i.e.

$$
\mathbf{x}_{/ \mathbf{B}_{\text {hex }}^{p}}^{\prime}=\mathbf{D}_{\text {hex }}^{p \rightarrow t} \mathbf{x}_{/ \mathbf{B}_{\text {hex }}^{p}} \rightarrow \mathbf{x}_{/ \mathbf{B}_{\text {hex }}^{t}}^{\prime}=\mathbf{C}_{\text {hex }}^{t \rightarrow p} \mathbf{x}_{/ \mathbf{B}_{\text {hex }}^{p}}
$$

\section{S1.2. Construction of the distortion, misorientation and correspondence matrices}

The crystallographic features of a twin model are determined by the choice of a supercell. This supercell defines a sub-lattice of the hexagonal lattice; and it is actually this sub-lattice that is linearly 
distorted by $\mathbf{D}^{p \rightarrow t}$; the atoms inside the supercell do not follow the same trajectories as those at the corners of the cells; they "shuffle". The supercell is formed by three crystallographic directions A, B, C defining a matrix

$$
\mathbf{B}_{\text {super }}^{p}=\left[\mathbf{B}_{\text {hex }}^{p} \rightarrow \mathbf{B}_{\text {super }}^{p}\right]=(\mathbf{A}, \mathbf{B}, \mathbf{C})_{/ \mathbf{B}_{\text {hex }}^{p}} .
$$

After distortion, the vectors of this basis are transformed into A', B', C' that define a new basis expressed in $\mathbf{B}_{\text {hex }}^{p}$ by the matrix $\mathbf{B}_{\text {super }}^{p^{\prime}}=\left(\mathbf{A}^{\prime}, \mathbf{B}^{\prime}, \mathbf{C}^{\prime}\right)_{/ \mathbf{B}_{\text {hex }}^{p}}=\left[\mathbf{B}_{\text {hex }}^{p} \rightarrow \mathbf{B}_{\text {super }}^{p^{\prime}}\right]$. When the vectors are expressed in the $\mathbf{B}_{\text {hex }}^{t}$, it takes the form $\mathbf{B}_{\text {super }}^{t}=\left(\mathbf{A}^{\prime}, \mathbf{B}^{\prime}, \mathbf{C}^{\prime}\right)_{/ \mathbf{B}_{\text {hex }}^{t}}=\left[\mathbf{B}_{\text {hex }}^{t} \rightarrow \mathbf{B}_{\text {super }}^{t}\right]$. As $\mathbf{B}_{\text {super }}^{p^{\prime}}$ and $\mathbf{B}_{\text {super }}^{t}$ express the same vectors, we get

$$
\left[\mathbf{B}_{\text {super }}^{\text {pu }} \rightarrow \mathbf{B}_{\text {super }}^{t}\right]=\mathbf{I}
$$

with I the identity matrix. Building a crystallographic model dedicated to a specific twin consists in finding the appropriate vectors $\mathbf{A}, \mathbf{B}, \mathbf{C}$ of the supercell and finding how they are transformed into $\mathbf{A}^{\mathbf{y}}$, B', C’. The three important matrices previously defined can be calculated from the supercell.

The distortion matrix is expressed in $\mathbf{B}_{\text {super }}^{p}$ by

$$
\mathbf{D}_{\text {super }}^{p \rightarrow t}=\left[\mathbf{B}_{\text {super }}^{p} \rightarrow \mathbf{B}_{\text {super }}^{p^{\prime}}\right]=\left[\mathbf{B}_{\text {super }}^{p} \rightarrow \mathbf{B}_{\text {hex }}^{p}\right]\left[\mathbf{B}_{\text {hex }}^{p} \rightarrow \mathbf{B}_{\text {super }}^{p \prime}\right]=\left(\mathbf{B}_{\text {super }}^{p}\right)^{-1} \mathbf{B}_{\text {super }}^{p \prime}
$$

As the distortion matrix is an active matrix; writing it in the basis $\mathbf{B}_{h e x}^{p}$ leads to

$$
\begin{gathered}
\mathbf{D}_{\text {hex }}^{p \rightarrow t}=\left[\mathbf{B}_{\text {hex }}^{p} \rightarrow \mathbf{B}_{\text {super }}^{p}\right] . \mathbf{D}_{\text {super }}^{p \rightarrow t}\left[\mathbf{B}_{\text {super }}^{p} \rightarrow \mathbf{B}_{\text {hex }}^{p}\right]=\mathbf{B}_{\text {super }}^{p}\left(\mathbf{B}_{\text {super }}^{p}\right)^{-1} \mathbf{B}_{\text {super }}^{p \prime}\left(\mathbf{B}_{\text {super }}^{p}\right)^{-1} \text {, i.e. } \\
\mathbf{D}_{\text {hex }}^{p \rightarrow t}=\mathbf{B}_{\text {super }}^{p \prime}\left(\mathbf{B}_{\text {super }}^{p}\right)^{-1}
\end{gathered}
$$

The misorientation matrix is $\mathbf{T}_{\text {hex }}^{p \rightarrow t}=\left[\mathbf{B}_{\text {hex }}^{p} \rightarrow \mathbf{B}_{\text {hex }}^{t}\right]=\left[\mathbf{B}_{\text {hex }}^{p} \rightarrow \mathbf{B}_{\text {super }}^{p \prime}\right]\left[\mathbf{B}_{\text {super }}^{p^{\prime}} \rightarrow\right.$ $\left.\mathbf{B}_{\text {super }}^{t}\right]\left[\mathbf{B}_{\text {super }}^{t} \rightarrow \mathbf{B}_{\text {hex }}^{t}\right]$, i.e.

$$
\mathbf{T}_{\text {hex }}^{p \rightarrow t}=\mathbf{B}_{\text {super }}^{p \prime}\left(\mathbf{B}_{\text {super }}^{t}\right)^{-1}
$$

The correspondence matrix is $\mathbf{C}_{\text {hex }}^{t \rightarrow p}=\left[\mathbf{B}_{\text {hex }}^{t} \rightarrow \mathbf{B}_{\text {hex }}^{p \prime}\right]=\left[\mathbf{B}_{\text {hex }}^{t} \rightarrow \mathbf{B}_{\text {hex }}^{p}\right]\left[\mathbf{B}_{\text {hex }}^{p} \rightarrow \mathbf{B}_{\text {hex }}^{p \prime}\right]$, i.e.

$\mathbf{C}_{\text {hex }}^{t \rightarrow p}=\mathbf{T}_{\text {hex }}^{t \rightarrow p} \mathbf{D}_{\text {hex }}^{p \rightarrow t}$, as found in equation S5. It can also be decomposed into $\mathbf{C}_{\text {hex }}^{t \rightarrow p}=\left[\mathbf{B}_{\text {hex }}^{t} \rightarrow \mathbf{B}_{\text {super }}^{t}\right]\left[\mathbf{B}_{\text {super }}^{t} \rightarrow \mathbf{B}_{\text {hex }}^{p \prime}\right]=\left[\mathbf{B}_{\text {hex }}^{t} \rightarrow \mathbf{B}_{\text {super }}^{t}\right]\left[\mathbf{B}_{\text {super }}^{t} \rightarrow \mathbf{B}_{\text {super }}^{p \prime}\right]\left[\mathbf{B}_{\text {super }}^{p \prime} \rightarrow \mathbf{B}_{\text {hex }}^{p \prime}\right]$

As the coordinates of the supercell are not changed by the distortion $\left[\mathbf{B}_{\text {hex }}^{p \prime} \rightarrow \mathbf{B}_{\text {super }}^{p \prime}\right]=$ $\left[\mathbf{B}_{\text {hex }}^{p} \rightarrow \mathbf{B}_{\text {super }}^{p}\right]=\mathbf{B}_{\text {super }}^{p}$, and by using S7, we get

$$
\mathbf{C}_{\text {hex }}^{t \rightarrow p}=\mathbf{B}_{\text {super }}^{t}\left(\mathbf{B}_{\text {super }}^{p}\right)^{-1}
$$

As the matrices $\mathbf{B}_{\text {super }}^{p}$ and $\mathbf{B}_{\text {super }}^{t}$ are constituted by the crystallographic directions forming the supercell, their values are integers. As the inverse of an integer matrix is a rational matrix, the correspondence matrix is a rational matrix. 


\section{S1.3. Obliquity correction}

It is usual in the crystallographic models of ferroelectrics to introduce an obliquity correction. This is a rotation with a small angle (few degrees) that is composed with a stretch distortion matrix in order to transform it into a simple shear matrix. An obliquity correction can be introduced to correct a small tilt on a plane and/or an small rotation of a direction. Here we need to introduce a general obliquity correction function $\mathbf{O b}\left(\boldsymbol{g}, \boldsymbol{g}^{\prime}, \boldsymbol{u}, \boldsymbol{u}^{\prime}\right)$. This function gives the rotation matrix noted $\mathbf{O b l}$ such that Obl. $\boldsymbol{g}=\boldsymbol{g}^{\prime}$ and $. \boldsymbol{u}=\boldsymbol{u}^{\prime}$. Let us consider a direction $\boldsymbol{u}$ and a plane $\boldsymbol{g}$ expressed in the hexagonal basis. Expressed in the orthonormal basis $\mathbf{B}_{\text {ortho }}$ they are $\boldsymbol{u}_{\boldsymbol{o}}=\mathbf{H}_{\text {hex }} \boldsymbol{u}$ and $\boldsymbol{g}_{\boldsymbol{o}}=\mathbf{H}_{\text {hex }}^{*} \boldsymbol{g}$. In this basis the plane $\boldsymbol{g}$ has the same coordinates as its normal direction $\boldsymbol{n}_{\boldsymbol{o}}$. A third direction defined by $\boldsymbol{l}_{\mathbf{0}}=$ $\boldsymbol{n}_{\boldsymbol{o}} \wedge \boldsymbol{u}_{\boldsymbol{o}}$ allows building another orthonormal basis $\mathbf{B}(\mathbf{g}, \boldsymbol{u})=\left(\frac{\boldsymbol{u}_{o}}{\left\|\boldsymbol{u}_{o}\right\|}, \frac{\boldsymbol{n}_{o}}{\left\|\boldsymbol{n}_{\boldsymbol{o}}\right\|}, \frac{\boldsymbol{l}_{\mathbf{0}}}{\left\|\boldsymbol{l}_{0}\right\|}\right)$. We build the orthonormal bases $\mathbf{B}(\boldsymbol{g}, \boldsymbol{u})$ and $\mathbf{B}\left(\boldsymbol{g}^{\prime}, \boldsymbol{u}^{\prime}\right)$. The obliquity rotation is

$$
\operatorname{Obl}\left(g, g^{\prime}, \boldsymbol{u}, \boldsymbol{u}^{\prime}\right)=\mathrm{B}\left(\boldsymbol{g}^{\prime}, \boldsymbol{u}^{\prime}\right)(\mathrm{B}(\boldsymbol{g}, \boldsymbol{u}))^{-1}
$$

It is a rotation matrix expressed in the orthonormal basis $\mathbf{B}_{\text {ortho }}$ that transforms $\mathbf{B}(\boldsymbol{g}, \boldsymbol{u})$ into $\mathbf{B}\left(\boldsymbol{g}^{\prime}, \boldsymbol{u}^{\prime}\right)$. This rotation should be compensated by its inverse in order to put in coincidence the plane $\boldsymbol{g}$ with the plane $\boldsymbol{g}^{\prime}$, and the direction $\boldsymbol{u}$ with the direction $\boldsymbol{u}^{\prime}$.

\section{S1.4. Definition of unconventional twinning}

We call conventional twin a twin whose lattice distortion is expressed by a simple shear matrix. The habit plane of these twins is the shear plane, which is also the plane maintained fully invariant by the shear distortion. This means that for two non-collinear directions $\boldsymbol{u}$ and $\boldsymbol{v}$ of the plane $\boldsymbol{g}$, i.e. such that $\boldsymbol{g} \cdot \boldsymbol{u}=\boldsymbol{g} \cdot \mathbf{v}=0$, are invariant by the distortion: $\mathbf{D}^{p \rightarrow t} \boldsymbol{u}=\boldsymbol{u}$ and $\mathbf{D}^{p \rightarrow t} \boldsymbol{v}=\boldsymbol{v}$. This implies that the dimension of the space formed by the kernel of $\mathbf{D}^{p \rightarrow t}-\mathbf{I}$ is such that

$$
\operatorname{Dim}\left(\operatorname{Ker}\left(\mathbf{D}^{p \rightarrow t}-\mathbf{I}\right)\right)=2
$$

If the plane $\boldsymbol{g}$ is invariant, it is untilted. Therefore, a consequence of the existence of an invariant plane is

$$
\boldsymbol{g}^{\prime}=\left(\mathbf{D}^{p \rightarrow t}\right)^{*} \boldsymbol{g}=\lambda \boldsymbol{g}
$$

which means that $\boldsymbol{g}$ is an eigenvector of $\left(\mathbf{D}^{p \rightarrow t}\right)^{*}$.

It should be noted that $\mathrm{S} 12 \Rightarrow \mathrm{S} 13$, but the reciprocal is not always true.

By noting the plane $\boldsymbol{g}$ by its Miller indices $\boldsymbol{g}=(h, k, l)$, and considering that the interplanar distance $d_{h k l}=\frac{1}{\|\boldsymbol{g}\|}$, we get

$$
\frac{1}{\lambda}=\frac{d^{\prime}{ }_{h k l}}{d_{h k l}}
$$


As the plane is invariant, the volume change is completely given by $1 / \lambda$. If $\lambda=1$, there is no volume change, the shear is called "simple shear". In the more general case, the shear is sometimes called "invariant plane strain” (IPS) and not "shear" in order to distinguish it from pure shear (stretch). To our knowledge, all the deformation twins reported in literature till now are simple shear.

In the manuscript, we call unconventional twin a twin defined by a distortion matrix for which a plane is untilted, but not invariant. Mathematically it means that the distortion matrix checks equation S13 but not equation S12. The untilted plane is transformed into a plane that is not equivalent to the initial one by any of the crystal symmetries; some of the directions contained in the plane are modified in length and/or angle. To our knowledge, unconventional twinning has never been reported till now.

S2. Unconventional $(212) \rightarrow(012)$ twinning mode built by obliquity correction of the $\left(58^{\circ}, \mathrm{a}+\right.$ 2b) prototype stretch twin

\section{S2.1. The $\left(58^{\circ}, a+2 b\right)$ prototype stretch twin}

The calculations were performed with Mathematica (see Supplementary Program Part A). This twin mode is also largely described by Cayron (2018); only its main characteristics are recalled here.

Let us use the letter "p" for the parent crystal, and "gr" for the $\sim\left(58^{\circ}, \mathbf{a}+2 \mathbf{b}\right)$ twins colored in "green" in the EBSD maps. The $p \rightarrow g r$ distortion is associated with the transformation $\mathbf{0} \mathbf{X}_{2} \rightarrow \mathbf{O} \mathbf{X}_{2}^{\prime}$, $\mathbf{O Y} \rightarrow$ $\mathbf{O} \mathbf{Y}^{\prime}, \mathbf{O G} \rightarrow \mathbf{O G}^{\prime}$ such that

- $\mathbf{O X}_{2}=[200]_{p}$ is parallel to the twin vector $\mathbf{O X}_{2}{ }^{\prime}=[\overline{1} 01]_{g r}$

- OY is invariant, $\mathbf{O Y}=[120]_{p}$ is equal to $\mathbf{O Y} \mathbf{Y}^{\prime}=[120]_{\mathrm{gr}}$

- $\mathbf{O G}=[\overline{1} 01]_{p}$ is parallel to the twin vector $\mathbf{O G}^{\prime}=[\overline{2} 00]_{g r}$

The vectors are here expressed by their hexagonal coordinates. The distortion matrix is thus ${ }^{28}$

$$
\mathbf{F}_{\text {hex }}^{p \rightarrow g r}=\left(\begin{array}{ccc}
\frac{\sqrt{1+\gamma^{2}}}{2} & \frac{2-\sqrt{1+\gamma^{2}}}{4} & \frac{\gamma^{2}-3}{2 \sqrt{1+\gamma^{2}}} \\
0 & 1 & 0 \\
0 & 0 & \frac{2}{\sqrt{1+\gamma^{2}}}
\end{array}\right)
$$

The values of the principal strains can be calculated in the cases of ideal hard-sphere packing and pure magnesium; they are $(-4.2 \%, 0,+4.4 \%)$ or $(-4.6 \%, 0,+4.8 \%)$, respectively.

The correspondence matrix is calculated by considering the vectors $\mathbf{0} \mathbf{X}_{2}, \mathbf{O Y}$, and $\mathbf{O G}$, and the vectors $\mathbf{O X}_{2}{ }^{\prime}, \mathbf{O} \mathbf{Y}^{\prime}$, and $\mathbf{O} \mathbf{G}^{\prime}$, in their respective hexagonal bases, i.e. by using the supercell $\left(\mathbf{O} \mathbf{X}_{2}, \mathbf{O Y}, \mathbf{O G}\right)$ :

$$
\mathbf{B}_{\text {super }}^{p}=\left(\begin{array}{ccc}
2 & 1 & -1 \\
0 & 2 & 0 \\
0 & 0 & 1
\end{array}\right) \text { and } \mathbf{B}_{\text {super }}^{g r}=\left(\begin{array}{ccc}
1 & 1 & -2 \\
0 & 2 & 0 \\
1 & 0 & 0
\end{array}\right)
$$

The expressions of the correspondence matrix in the direct and reciprocal space are:

$$
\mathbf{C}_{\text {hex }}^{g r \rightarrow p}=\mathbf{B}_{\text {super. }}^{g r}\left(\mathbf{B}_{\text {super }}^{p}\right)^{-1}=\left(\begin{array}{ccc}
\frac{1}{2} & \frac{1}{4} & -\frac{3}{2} \\
0 & 1 & 0 \\
\frac{1}{2} & -\frac{1}{4} & \frac{1}{2}
\end{array}\right) \text {, and }
$$




$$
\left(\mathbf{C}_{\text {hex }}^{g r \rightarrow p}\right)^{*}=\left(\mathbf{C}_{\text {hex }}^{g r \rightarrow p}\right)^{-T}=\left(\begin{array}{ccc}
\frac{1}{2} & 0 & -\frac{1}{2} \\
\frac{1}{4} & 1 & \frac{1}{4} \\
\frac{3}{2} & 0 & \frac{1}{2}
\end{array}\right)
$$

The misorientation matrix is given by equation S5:

$$
\mathbf{T}_{\text {hex }}^{p \rightarrow t}=\mathbf{D}_{\text {hex }}^{p \rightarrow t}\left(\mathbf{C}_{\text {hex }}^{t \rightarrow p}\right)^{-1}=\left(\begin{array}{ccc}
\frac{1}{\sqrt{1+\gamma^{2}}} & 0 & \frac{\gamma}{\sqrt{1+\gamma^{2}}} \\
0 & 1 & 0 \\
-\frac{\gamma}{\sqrt{1+\gamma^{2}}} & 0 & \frac{1}{\sqrt{1+\gamma^{2}}}
\end{array}\right)
$$

which is a rotation of angle $\operatorname{ArcCos}\left(\frac{1}{\sqrt{1+\gamma^{2}}}\right)=\operatorname{ArcTan}(\gamma)$, that is equal to $58.5^{\circ}$ for hard-sphere packing and $58.4^{\circ}$ for magnesium.

Some correspondences between some planes and directions of the parent and its twins calculated from the correspondence matrices in equation S17 are interesting to interpret the EBSD map. They are given in Table S1.

\begin{tabular}{|lllll|}
\hline Parent & $\rightarrow$ & Twin \\
\hline \multicolumn{5}{|c|}{ Planes } \\
\hline$(\mathbf{2 1 2})$ & $\in\{\mathbf{1 2} \overline{\mathbf{3}} \mathbf{2}\}$ & $\rightarrow$ & $(\mathbf{0 2 4})$ & $\in\{\mathbf{0 2} \overline{\mathbf{2}} \mathbf{4}\}$ \\
\hline$(004)$ & $\in\{0004\}$ & $\rightarrow$ & $(\overline{2} 12)$ & $\in\{\overline{\mathbf{2}} 112\}$ \\
\hline \multicolumn{5}{|c|}{ Directions } \\
\hline$[\mathbf{0} \overline{\mathbf{2}} \mathbf{1}]$ & $\in \frac{\mathbf{1}}{\mathbf{3}}\langle\mathbf{2 2} \overline{\mathbf{4}} \mathbf{3}\rangle$ & $\rightarrow$ & {$[\overline{\mathbf{2}} \mathbf{2} 1]$} & $\in \frac{\mathbf{1}}{\mathbf{3}}\langle\mathbf{2 2} \overline{\mathbf{4}} \mathbf{3}\rangle$ \\
\hline$[200]$ & $\in \frac{1}{3}\langle 22 \overline{4} 0\rangle$ & $\rightarrow$ & {$[101]$} & $\in \frac{1}{3}\langle 11 \overline{2} 3\rangle$ \\
\hline$[\overline{1} 01]$ & $\in \frac{1}{3}\langle 11 \overline{2} 3\rangle$ & $\rightarrow$ & {$[\overline{2} 00]$} & $\in \frac{1}{3}\langle 22 \overline{4} 0\rangle$ \\
\hline$[120]$ & $\in\langle 01 \overline{1} 0\rangle$ & $\rightarrow$ & {$[120]$} & $\in\langle 01 \overline{1} 0\rangle$ \\
\hline
\end{tabular}

Table S1 Correspondence between some planes and between some directions established by the $\left(58^{\circ}\right.$, a) stretch twin. The families of their equivalent directions/planes are indicated by using the fourindex Miller-Bravais notations. The plane $\mathbf{g}_{0}=(212)_{p}$ and the direction $\mathbf{u}_{0}=[0 \overline{2} 1]_{p}$ of the parent crystal (in bold) will be used to build the model of the green twins.

From this table, we tried two different approaches to build a model that could explain the green twins observed experimental EBSD maps. The first approach was the most intuitive one; it is based on the 
fact that the direction $\mathbf{O Y}=[120]_{p}$ is invariant in the stretch twin model (see Table S1). However, after many attempts, this way was given up because all the habit planes we could predict contain the OY direction, which is not in agreement with the observations. A dissymmetry should be introduced in the system. The second approach was less intuitive; but it was revealed to fit perfectly with the observations, even for small details that were not noticed at the beginning. It is based on the correspondence between the $(212)_{p}$ and $(012)_{g r}$ planes, and between the $[0 \overline{2} 1]_{p}$ and $[\overline{2} \overline{2} 1]_{g r}$ directions (Supporting Table S1). The model, described in the next section, introduces an obliquity correction such that the plane $\boldsymbol{g}_{0}=(212)_{p}$ becomes untilted and the direction $\boldsymbol{u}_{0}=[0 \overline{2} 1]_{p}$ invariant.

\section{S2.2. Unconventional twin derived from the $\left(58^{\circ}, a+2 b\right)$ stretch twin prototype}

The calculations were performed with Mathematica (see Supplementary Program Part B).

The EBSD map shows that the habit plane of the green twin is not invariant; it is the plane $(212)_{p}$ transformed into the plane $(012)_{g r}$. These two planes are not equivalent. The modification of this plane comes from the transformation of the directions it contains, i.e. $[\overline{101}]_{p}$ is transformed into $[\overline{2} 00]_{g r}$, and $[0 \overline{2} 1]_{p}$ is transformed into $[\overline{2} \overline{2} 1]_{g r}$. The transformation of the direction $[\overline{1} 01]_{p}$ occurs by a stretch of $\frac{2}{\sqrt{1+\gamma^{2}}} \approx 1.04$. There is no stretch for the $[0 \overline{2} 1]_{p}$ direction because it is equivalent to $[\overline{2} \overline{2} 1]_{g r}$. In addition, the angle formed by the pairs $\alpha_{p}=\left([\overline{1} 01]_{p},[0 \overline{2} 1]_{p}\right)=\operatorname{ArcCos}\left(\frac{\gamma^{2}-1}{\sqrt{4+5 \gamma^{2}+\gamma^{4}}}\right)$ $\approx 70.56^{\circ}$ is slightly reduced to become that the angle between the pair $\alpha_{\mathrm{t}}=\left([0 \overline{2} 1]_{g r},[\overline{2} \overline{2} 1]_{g r}\right)=$ $\operatorname{Arc} \operatorname{Cos}\left(\frac{1}{\sqrt{4+\gamma^{2}}}\right) \approx 67.15^{\circ}$. The stretch of the $[\overline{1} 01]_{p}$ direction $(+4 \%)$ and the angular distortion of the plane $\left(-3^{\circ}\right)$ are quite small. We noticed that the planar transformation $(212)_{p} \rightarrow(012)_{g r}$ can be explained by the displacements of the atoms located in the upper layer $l=1 / 3$ of the plane $(212)_{p}$ as described in Figure S4.

Even if the direction $\boldsymbol{u}_{\mathbf{0}}=[0 \overline{2} 1]_{p}$ is not stretched, the prototype twin induces a slight rotation of angle $\xi_{u}$ of this direction. This is this rotation that should be compensated in order to build the model. The rotation angle $\xi_{u}$ can be calculated by working in the orthonormal basis; it is the angle between $\mathbf{H}_{\text {hex }} \boldsymbol{u}_{\mathbf{0}}$ and $\mathbf{F}_{\text {ortho }}^{p \rightarrow t} . \mathbf{H}_{\text {hex }} \boldsymbol{u}_{\mathbf{0}}$, with $\mathbf{F}_{\text {ortho }}^{p \rightarrow t}$ the inverse of the transpose of the matrix S15. The calculations show that

$$
\xi_{u}=\operatorname{ArcCos}\left(\frac{-1+3 \gamma^{2}+3 \sqrt{1+\gamma^{2}}}{\sqrt{1+\gamma^{2}}\left(4+\gamma^{2}\right)}\right)
$$

For a hard-sphere packing ratio $\gamma=\sqrt{\frac{8}{3}}$, the obliquity is $\xi_{u}=\operatorname{ArcCos}\left(\frac{99+21 \sqrt{33}}{220}\right) \approx 3.29^{\circ}$. 
The stretch prototype twin also induces a rotation of the plane $\boldsymbol{g}_{\mathbf{0}}=(212)_{p}$. The rotation angle $\xi_{g}$ is the angle between $\mathbf{H}_{\text {hex }}^{*} \boldsymbol{g}_{\mathbf{0}}$ and $\left(\mathbf{F}_{\text {ortho }}^{p \rightarrow t}\right)^{*} \mathbf{H}_{\text {hex }}^{*} \boldsymbol{g}_{\mathbf{0}}$, with $\left(\mathbf{F}_{\text {ortho }}^{p \rightarrow t}\right)^{*}$ the inverse of the transpose of the matrix S15. The calculations show that

$$
\xi_{g}=\operatorname{ArcCos}\left(\frac{3+\gamma^{2}\left(3+2 \sqrt{1+\gamma^{2}}\right)}{\sqrt{\left(1+\gamma^{2}\right)\left(3+\gamma^{2}\right)\left(3+7 \gamma^{2}\right)}}\right)
$$

For a hard-sphere packing ratio $\gamma=\sqrt{\frac{8}{3}}$, the obliquity is $\xi_{g}=\operatorname{ArcCos}\left(\frac{16+3 \sqrt{33}}{\sqrt{1105}}\right) \approx 1.24^{\circ}$.

In order to correct in one shot the two obliquities $\xi_{u}$ and $\xi_{g}$ and rotate the stretch prototype twin such that the direction $\boldsymbol{u}_{\mathbf{0}}=[0 \overline{2} 1]_{p}$ becomes invariant and the plane $\boldsymbol{g}_{\mathbf{0}}=(212)_{p}$ becomes untilted, the general obliquity correction function $\mathbf{0 b l}\left(\boldsymbol{g}, \boldsymbol{g}^{\prime}, \boldsymbol{u}, \boldsymbol{u}^{\prime}\right)$ described in section S1.3 is used with $\boldsymbol{g}=(212)_{p}, \boldsymbol{g}^{\prime}=\left(\mathbf{F}_{\text {hex }}^{p \rightarrow t}\right)^{*} \boldsymbol{g}, \boldsymbol{u}=[0 \overline{2} 1]_{p}, \boldsymbol{u}^{\prime}=\mathbf{F}_{\text {hex }}^{p \rightarrow g r} \boldsymbol{u}$. The result expressed as a function of $\gamma$ is too long to be written here, even by writing separately each of its nine components. The reader can however see the result in Part B of the Supplementary Program.

In the special case of a hard-sphere packing ratio $\gamma=\sqrt{\frac{8}{3}}$, the approximate numerical value of the obliquity matrix is:

$$
\mathbf{0}_{\boldsymbol{g}, \boldsymbol{u}}=\mathbf{O b l}\left(\boldsymbol{g}, \boldsymbol{g}^{\prime}, \boldsymbol{u}, \boldsymbol{u}^{\prime}\right) \approx\left(\begin{array}{ccc}
0.9764 & 0.0452 & -0.0392 \\
-0.0443 & 1.0212 & 0.0423 \\
0.0231 & -0.0227 & 0.9991
\end{array}\right)
$$

The obliquity angle is $\operatorname{ArcCos}\left(\frac{1}{440}(-121+21 \sqrt{33})+\frac{803+369 \sqrt{33}}{88 \sqrt{1105}}\right) \approx 3.33^{\circ}$

The distortion matrix corrected of the obliquity is

$$
\mathbf{D}_{\text {hex }}^{p \rightarrow g r}=\mathbf{0}_{\boldsymbol{g}, \boldsymbol{u}}^{-\mathbf{1}} \cdot \mathbf{F}_{\text {hex }}^{p \rightarrow g r}
$$

Despite the very long analytical expression of the general form of the obliquity matrix $\mathbf{R}$, the distortion corrected from this obliquity can be calculated and simplified. The analytical expressions of the nine components $\mathbf{D}_{i j}$ of $\mathbf{D}_{\text {hex }}^{p \rightarrow g r}$ are: 


$$
\begin{aligned}
& \mathbf{D}_{11}=\frac{\mathrm{A}}{6+2 \gamma^{2}} \\
& \mathbf{D}_{12}=\frac{3\left(\gamma^{2}-3\right)}{4 \mathrm{~A}} \\
& \mathbf{D}_{13}=\frac{3\left(\gamma^{2}-3\right)}{2 \mathrm{~A}} \\
& \mathbf{D}_{21}=\frac{3-2 \gamma^{2}-\gamma^{4}+\mathrm{A}}{12+7 \gamma^{2}+\gamma^{4}} \\
& \mathbf{D}_{22}=\frac{-9+4 \gamma^{4}+5 \mathrm{~A}+\gamma^{2}(15+\mathrm{A})}{2\left(4+\gamma^{2}\right) \mathrm{A}} \\
& \mathbf{D}_{23}=\frac{4 \gamma^{4}-\gamma^{2}(-15+\mathrm{A})-3(3+\mathrm{A})}{\left(4+\gamma^{2}\right) \mathrm{A}} \\
& \mathbf{D}_{31}=\frac{-3+2 \gamma^{2}+\gamma^{4}-\mathrm{A}}{2\left(12+7 \gamma^{2}+\gamma^{4}\right)} \\
& \mathbf{D}_{32}=\frac{-45-59 \gamma^{4}-7 \gamma^{6}+57 \mathrm{~A}+\gamma^{2}(-129+13 \mathrm{~A})}{4\left(36+105 \gamma^{2}+52 \gamma^{4}+7 \gamma^{6}\right)} \\
& \mathbf{D}_{33}=\frac{27+45 \gamma^{4}+7 \gamma^{6}+57 \mathrm{~B}+\gamma^{2}(81+13 \mathrm{~A})}{2\left(36+105 \gamma^{2}+52 \gamma^{4}+7 \gamma^{6}\right)}
\end{aligned}
$$

with $A=\sqrt{\left(3+\gamma^{2}\right)\left(3+7 \gamma^{2}\right)}$

It is checked that this distortion matrix $\mathbf{D}_{\text {hex }}^{p \rightarrow g r}$ maintains invariant the direction $\boldsymbol{u}_{\mathbf{0}}=[0 \overline{2} 1]_{p}$ and that $\left(\mathbf{D}_{\text {hex }}^{p \rightarrow t}\right)^{*}$ maintains untilted the plane $\boldsymbol{g}_{\mathbf{0}}=(212)_{p}$.

For the ideal hard-sphere packing ratio, the distortion matrix takes the value

$$
\begin{aligned}
& \mathbf{D}_{\text {hex }}^{p \rightarrow g r}=\left(\begin{array}{ccc}
\frac{\sqrt{\frac{65}{17}}}{2} & -\frac{3}{4 \sqrt{1105}} & -\frac{3}{2 \sqrt{1105}} \\
-\frac{1}{4}+\frac{3 \sqrt{\frac{13}{85}}}{4} & \frac{23}{40}+\frac{107}{8 \sqrt{1105}} & -\frac{17}{20}+\frac{107}{4 \sqrt{1105}} \\
\frac{1}{8}-\frac{3 \sqrt{\frac{13}{85}}}{8} & -\frac{23}{80}+\frac{33 \sqrt{\frac{5}{221}}}{16} & \frac{17}{40}+\frac{33 \sqrt{\frac{5}{221}}}{8}
\end{array}\right) \\
& \approx\left(\begin{array}{ccc}
0.9777 & -0.0226 & -0.0451 \\
0.0433 & 0.9774 & -0.0453 \\
-0.0217 & 0.0227 & 1.0455
\end{array}\right)
\end{aligned}
$$

As $\mathbf{D}_{\text {hex }}^{p \rightarrow g r}$ differs from $\mathbf{F}_{\text {hex }}^{p \rightarrow g r}$ only by the obliquity correction, the correspondence matrix given by equation $\mathbf{S} 17$ is not affected. The distortion $\mathbf{D}_{\text {hex }}^{p \rightarrow g r}$ is unconventional as the untilted plane (212) which is also the habit plane of the green twin, is not fully invariant but transformed into the plane $(012)_{g r}$. The modes of plasticity required to accommodate this deformation are not the subject of the 
paper, but it is hoped that deeper TEM investigations and molecular dynamics simulations can bring important elements of responses.

We have seen in section S2.1 that the rotation matrix $\mathbf{T}_{\text {hex }}^{p \rightarrow g r}$ between the parent and the green twin associated with the stretch distortion $\mathbf{F}_{\text {hex }}^{p \rightarrow g r}$ is a rotation of axis $\mathbf{O Y}=[120]_{\text {hex }}$ and of angle $\theta_{F}=$ $\operatorname{Arc} \operatorname{Cos}\left(\frac{1}{\sqrt{1+\gamma^{2}}}\right)$, that is $58.5^{\circ}$ for hard-sphere packing, $58.4^{\circ}$ for magnesium. Now, if instead of the stretch prototype $\mathbf{F}_{h e x}^{p \rightarrow g r}$, the distortion $\mathbf{D}_{\text {hex }}^{p \rightarrow g r}$ applies, the orientation of the twinned crystal is slightly modified. The new expression of orientation matrix $\mathbf{T}_{h e x}^{p \rightarrow g r}$ between the twin and the parent is obtained by using the distortion matrix and the correspondence matrix in equation S5; it is calculated in part B of the Supplementary Program:

$$
\mathbf{T}_{\text {hex }}^{p \rightarrow g r}=\left(\begin{array}{ccc}
\sqrt{\frac{3+\gamma^{2}}{3+7 \gamma^{2}}} & \frac{-3+2 \gamma^{2}}{\mathrm{~A}} & \frac{6 \gamma^{2}}{\mathrm{~A}} \\
\frac{2\left(3-\gamma^{2}\left(2+\gamma^{2}\right)+A\right)}{\left(4+\gamma^{2}\right) \mathrm{A}} & \frac{-6+13 \gamma^{2}+3 \gamma^{4}+2 \mathrm{~A}}{\left(4+\gamma^{2}\right) \mathrm{A}} & \frac{2 \gamma^{2}\left(9+\gamma^{2}-\mathrm{A}\right)}{\left(4+\gamma^{2}\right) \mathrm{A}} \\
\frac{18}{3+7 \gamma^{2}-5 \mathrm{~A}} & \frac{9\left(12+\gamma^{2}\right)}{9+7 \gamma^{4}+21 \mathrm{~A}+4 \gamma^{2}(6+\mathrm{A})} & \frac{24 \gamma^{4}+7 \gamma^{6}+12 \mathrm{~A}+\gamma^{2}(9-2 A)}{\left(3+7 \gamma^{2}\right)\left(12+7 \gamma^{2}+\gamma^{4}\right)}
\end{array}\right)
$$

with $A=\sqrt{\left(3+\gamma^{2}\right)\left(3+7 \gamma^{2}\right)}$

The rotation angle is

$$
\theta_{D}=\operatorname{ArcCos}\left(\frac{9+9 \gamma^{2}+2 \gamma^{4}-\mathrm{A}}{\left(4+\gamma^{2}\right) \mathrm{A}}\right)
$$

The rotation axis is a complex form of the packing ratio $\gamma$; it slightly deviates from the axis $\mathbf{O Y}=$ $[120]_{\text {hex. }}$ In the case of the ideal hard-sphere packing ratio, the rotation angle is

$\theta_{D}=\operatorname{ArcCos}\left(-\frac{3}{20}+\frac{\sqrt{\frac{85}{13}}}{4}\right) \approx 60.71^{\circ}$, and the axis is $\left[1,2, \frac{3}{(31+\sqrt{1105})}\right]_{\text {hex }} \approx[1,2,0.047]_{\text {hex }}$. For magnesium the angle is $\theta_{D} \approx 60.76^{\circ}$, and the axis is $\approx[1,2,0.051]_{\text {hex }}$. Consequently, a careful examination of the rotation angle of the misorientation between the twin and its parent permits to know whether this twin directly comes from the prototype stretch distortion $\mathbf{F}_{\text {hex }}^{p \rightarrow g r}$ or from its derived obliquity-corrected form, i.e. $\mathbf{D}_{h e x}^{p \rightarrow g r}$. In the former case the misorientation angle is close $58^{\circ}$ and in the latter case is close to $61^{\circ}$. Both forms exist in the EBSD maps of Figure1 and Figure S1. The other method to distinguish the twin generated by distortion $\mathbf{F}_{\text {hex }}^{p \rightarrow g r}$ from the one generated by $\mathbf{D}_{\text {hex }}^{p \rightarrow g r}$ consists in considering the $\langle 201\rangle$ directions. All are rotated by the distortion $\mathbf{F}_{\text {hex }}^{p \rightarrow g r}$ whereas the distortion matrix $\mathbf{D}_{\text {hex }}^{p \rightarrow g r}$ maintains the direction $\boldsymbol{u}_{\mathbf{0}}=[0 \overline{2} 1]_{p}$ invariant.

A rotation equivalent to $\mathbf{T}_{h e x}^{p \rightarrow g r}$ that has $\boldsymbol{u}_{\mathbf{0}}$ for rotation axis is found by using a 6-fold rotation symmetry. In the basis $\mathbf{B}_{\text {hex }}$, and noted by its Seitz symbol, this symmetry is

$$
\mathbf{6}_{001}^{-}=\left(\begin{array}{ccc}
0 & 1 & 0 \\
-1 & 1 & 0 \\
0 & 0 & 1
\end{array}\right)
$$


The equivalent rotation between the twin and the parent crystal is $\mathbf{T}_{h e x}^{p \rightarrow g r} \cdot \mathbf{6}_{001}^{-}$. This rotation matrix is explicitly written in PartB of the Supplementary Program. The rotation angle is

$$
\theta_{D}=\operatorname{ArcCos}\left(\frac{6-\gamma^{2}}{2 \mathrm{~A}}\right)
$$

In the case of hard-sphere packing, this angle is $\theta_{D}=\operatorname{ArcCos}\left(\sqrt{\frac{5}{221}}\right) \approx 81.35^{\circ}$. It was checked that the rotation axis is indeed $\boldsymbol{u}_{\mathbf{0}}=[0 \overline{2} 1]_{p}$, independently of the packing ratio $\gamma$.The commercial EBSD programs do not give all the equivalent rotations but only the disorientation, i.e. the rotation that among all the equivalent rotations has the lowest angle. The present example shows that this choice is sometimes not well adapted, as the rotation axis of $\mathbf{T}_{\text {hex }}^{p \rightarrow g r}$ is complex, even if close to [120], whereas that of $\mathbf{T}_{h e x}^{p \rightarrow g r} \cdot \mathbf{6}_{001}^{-}$is simply a rotation around the [0릴 direction.

\section{S3. Unconventional $(012) \rightarrow(212)$ twinning mode by obliquity correction of the $\left(86^{\circ}\right.$, a) twin}

The experimental EBSD maps show that the extension "yellow" twins are often co-formed with the "green" twins and constitute green-yellow "stripes" as that in the green rectangle of Figure1a. In the EBSD map acquired in the cross-section B, the yellow twins can also appear orange or red, as shown in the Figure S1. The striking point is that these the "yellow" twins are conventional twins of the parent "grey" crystal: their habit plane is the plane $(212)_{p}$, and this plane is common to both the parent and "yellow" crystal. The misorientation between the "yellow" twins and the parent "grey" crystal experimentally measured from the EBSD maps is a rotation of $48^{\circ}$ around an axis close to a $\langle 241\rangle$ direction, as shown in Figure1b,c. To the best of our knowledge this twin has never been reported or predicted; which means that, even if conventional, there is not yet crystallographic model for it. In order to build such a model, additional information is required. We noticed that the misorientation between the yellow twins and the green twins is close to $\left(86^{\circ}, \mathbf{a}\right)$, with an interface plane close to $\{102\}$, which means that the yellow and green twins are linked by an kind of extension twin relation, or a twinning relation close to that one.

The crystallographic model of $\left(86^{\circ}\right.$, a) extension twinning in hcp metals was proposed by correcting the obliquity of a $\left(90^{\circ}\right.$, a) prototype stretch twin to maintain a plane $\{102\}$ untilted (Cayron, 2017a). The correspondence matrix written in the reciprocal space shows that among the five other equivalent $\{102\}$ planes, one is also transformed into another $\{102\}$ plane (by conjugation), and the four other ones are transformed into $\{212\}$ planes. Some of these four $\{102\}$ planes transformed into $\{212\}$ planes are only slightly tilted during the extension twinning. Thus, it is possible, by adding an obliquity correction to a conventional extension twin, to change the conventional extension twin into an unconventional twin that transforms a $\{102\}$ plane into a $\{212\}$ plane without tilt. The green twin transforms a $\{212\}_{p}$ plane into a $\{102\}_{g r}$ plane, and the yellow twin would transform back this $\{102\}_{g r}$ plane into a $\{212\}_{p}$ plane, such that the yellow twin would leave invariant the $\{212\}_{p}$ plane 
of the parent crystal, i.e. $\{212\}_{p}=\{212\}_{y e}$, as observed in the EBSD maps. Before detailing the obliquity correction that will be applied to the conventional extension twin, let us determine the appropriate reference frame that should be used to express the extension twinning distortion matrix in order to be composed with the green twin.

\section{S3.1. The conventional $\left(86^{\circ}\right.$, a) twin in an adequate basis}

The calculations were performed with Mathematica (see Supplementary Program Part C).

In order to build the unconventional yellow twin derived from a conventional $\left(86^{\circ}, \boldsymbol{a}\right)$ extension twin, we have to quickly recall some the crystallographic details of this twin. The $\left(86^{\circ}, \boldsymbol{a}\right)$ extension twin described in the paper ${ }^{21}$ is an extension twin on the plane $(0 \overline{1} 2)_{p}$. This twin was shown to derive from a stretch prototype, called $\left(90^{\circ}, \boldsymbol{a}\right)$ twin. Most of the calculations (Cayron, 2017a) were done by assuming an ideal hard-sphere packing ratio in order to determine the continuous form of the distortion. The calculations related to the general case depending on $\gamma$ were not explicitly detailed. Let us present them now. The distortion matrix associated with the $\left(90^{\circ}, \boldsymbol{a}\right)$ twin is

$$
\mathbf{U}_{\text {hex }}^{p \rightarrow t}=\left(\begin{array}{ccc}
1 & 0 & 0 \\
0 & \frac{\gamma}{\sqrt{3}} & 0 \\
0 & 0 & \frac{\sqrt{3}}{\gamma}
\end{array}\right)
$$

This $\left(90^{\circ}, \boldsymbol{a}\right)$ stretch prototype twin induces a rotation of the plane $\boldsymbol{g}=(0 \overline{1} 2)_{p}$ around the axis $[100]_{p}$. This rotation $\mathbf{R}_{\boldsymbol{g}}$ has for rotation angle $\xi_{g}$ that can be calculated by working in the orthonormal basis; it is the angle between $\mathbf{H}_{\text {hex }}^{*} \boldsymbol{g}$ and $\left(\mathbf{U}_{\text {ortho }}^{p \rightarrow t}\right)^{*}$. $\mathbf{H}_{\text {hex }}^{*} \boldsymbol{g}$, with $\left(\mathbf{U}_{\text {ortho }}^{p \rightarrow t}\right)^{*}$ the inverse of the transpose of the matrix S29. The calculations computed in Part C of the Supplementary

Program show that

$$
\xi_{g}=\operatorname{ArcCos}\left(\frac{2 \sqrt{3} \gamma}{3+\gamma^{2}}\right)
$$

After the obliquity correction, the distortion matrix becomes

$$
\mathbf{E}_{\text {hex }}^{p \rightarrow t}=\mathbf{R}_{\boldsymbol{g}}^{-\mathbf{1}} \cdot \mathbf{U}_{\text {hex }}^{p \rightarrow t}=\left(\begin{array}{ccc}
1 & -\frac{3-\gamma^{2}}{2\left(3+\gamma^{2}\right)} & \frac{3-\gamma^{2}}{3+\gamma^{2}} \\
0 & \frac{2 \gamma^{2}}{3+\gamma^{2}} & \frac{2\left(3-\gamma^{2}\right)}{3+\gamma^{2}} \\
0 & -\frac{3-\gamma^{2}}{2\left(3+\gamma^{2}\right)} & \frac{6}{3+\gamma^{2}}
\end{array}\right)
$$

For the ideal hard-sphere packing ratio, the obliquity is $\xi_{g}=\operatorname{ArcCos}\left(\frac{12 \sqrt{2}}{17}\right) \approx 3.37^{\circ}$, and the obliquity-corrected distortion matrix becomes

$$
\mathbf{E}_{\text {hex }}^{p \rightarrow t}=\left(\begin{array}{ccc}
1 & -\frac{1}{34} & \frac{1}{17} \\
0 & \frac{16}{17} & \frac{2}{17} \\
0 & -\frac{1}{34} & \frac{18}{17}
\end{array}\right)
$$


The distortion matrix S32 generates the conventional $\left(86^{\circ}\right.$, a) twin for which the invariant plane is $(0 \overline{1} 2)_{p}$. In order to continue working with coherent coordinates in the system formed by the "green", "yellow" and "grey" crystals, we need to use an extension twin such that, once combined with the green twin distortion S23, it yields a conventional twin on the $(212)_{p}$ plane. A hexagonal symmetry is thus introduced; its choice will be justified a posteriori by the internal coherency of the calculations and by the perfect agreement with the experimental EBSD observations. This internal symmetry noted by its Seitz symbol is

$$
\mathbf{2}_{110}=\left(\begin{array}{ccc}
0 & 1 & 0 \\
1 & 0 & 0 \\
0 & 0 & -1
\end{array}\right)
$$

It allows establishing the distortion matrix of the $(102)_{p}$ extension twin from that of the $(0 \overline{1} 2)_{p}$ extension twin given in equation S32:

$$
\mathbf{E}_{\text {hex }}^{g r \rightarrow y e}=\left(\mathbf{2}_{110}\right)^{-1} \mathbf{E}_{\text {hex }}^{p \rightarrow t} \mathbf{2}_{110}=\left(\begin{array}{ccc}
\frac{2 \gamma^{2}}{3+\gamma^{2}} & 0 & -\frac{2\left(3-\gamma^{2}\right)}{3+\gamma^{2}} \\
-\frac{3-\gamma^{2}}{2\left(3+\gamma^{2}\right)} & 1 & -\frac{3-\gamma^{2}}{\left(3+\gamma^{2}\right)} \\
\frac{3-\gamma^{2}}{2\left(3+\gamma^{2}\right)} & 0 & \frac{6}{3+\gamma^{2}}
\end{array}\right)
$$

To be clearer, we have used in equation S34 a notation that specifies that the parent crystal is the green grain and that the yellow grains are linked to it by an extension twin (even if not yet corrected by the obliquity). Indeed, the parent index "p" is here "gr" and the twin index "t" is "gr".

The correspondence matrices in the direct and reciprocal spaces are

$$
\begin{gathered}
\mathbf{C}_{\text {hex }}^{y e \rightarrow g r}=\mathbf{C}_{\text {hex }}^{y e \rightarrow g r} \mathbf{2}_{110}=\left(\begin{array}{ccc}
-\frac{1}{2} & 1 & 1 \\
0 & 0 & 2 \\
\frac{1}{2} & 0 & 0
\end{array}\right) \\
\text { and }\left(\mathbf{C}_{\text {hex }}^{y e \rightarrow g r}\right)^{*}=\left(\begin{array}{ccc}
0 & 1 & 0 \\
0 & -\frac{1}{2} & \frac{1}{2} \\
2 & 1 & 0
\end{array}\right)
\end{gathered}
$$

And the misorientation matrix is

$$
\mathbf{T}_{\text {hex }}^{g r \rightarrow y e}=\left(\mathbf{2}_{110}\right)^{-1} \mathbf{T}_{\text {hex }}^{g r \rightarrow y e}=\left(\begin{array}{ccc}
0 & 1-\frac{6}{3+\gamma^{2}} & \frac{4 \gamma^{2}}{3+\gamma^{2}} \\
1 & -\frac{3}{3+\gamma^{2}} & \frac{2 \gamma^{2}}{3+\gamma^{2}} \\
0 & \frac{3}{3+\gamma^{2}} & -1+\frac{6}{3+\gamma^{2}}
\end{array}\right)
$$

In the case of hard-sphere packing the distortion and orientation matrices take rational values:

$$
\mathbf{E}_{\text {hex }}^{g r \rightarrow y e}=\left(\begin{array}{ccc}
\frac{16}{17} & 0 & -\frac{2}{17} \\
-\frac{1}{34} & 1 & -\frac{1}{17} \\
\frac{1}{34} & 0 & \frac{18}{17}
\end{array}\right) \text { and } \mathbf{T}_{h e x}^{g r \rightarrow y e}=\left(\begin{array}{ccc}
0 & -\frac{1}{17} & \frac{32}{17} \\
1 & -\frac{9}{17} & \frac{16}{17} \\
0 & \frac{9}{17} & \frac{1}{17}
\end{array}\right)
$$


Now that the appropriate basis is found to express the conventional extension twin, the additional obliquity correction required to get the planar distortion (012) $\rightarrow$ (212) without tilt can be determined.

\section{S3.2. The unconventional twin derived from the $\left(86^{\circ}, a\right)$ twin prototype}

The calculations were performed with Mathematica (see Supplementary Program Part D).

The extension twin S34 leaves invariant the plane $(102)_{g r}$ and the direction $[\overline{2} \overline{2} 1]_{g r}$, and it transforms the plane $(012)_{g r}$ into the plane (212) $)_{y e}$ by the correspondence matrix S35, but this plane is tilted. Now, we will build by obliquity correction of the conventional extension twin S34 an unconventional twin such that the plane (012) gr is transformed into the plane (212) ye without tilt, and such that the direction $[\overline{2} \overline{2} 1]_{g r}$ becomes invariant. This twin, when composed with the unconventional "green" twin, will give a conventional twin relatively to the "grey” parent crystal. In order to determine the obliquity matrix, one could directly apply the general function S21, but we noticed that correcting the obliquity of the plane $\mathbf{g}=(012)_{g r}$ is sufficient to also correct the obliquity of the direction $[\overline{2} \overline{2} 1]_{g r}$, as detailed as follows.

The tilt $\xi_{g}$ of the plane $\mathbf{g}=(012)_{g r}$ by the conventional distortion matrix $\mathbf{E}_{\text {hex }}^{g r \rightarrow y e}$ can be calculated by working in the orthonormal basis; it is the angle between $\mathbf{H}_{\text {hex }}^{*} \boldsymbol{g}$ and $\mathbf{H}_{\text {hex }}^{*}\left(\mathbf{E}_{\text {hex }}^{\text {gr } \rightarrow y e}\right)^{*} \boldsymbol{g}$, with $\left(\mathbf{E}_{\text {hex }}^{g r \rightarrow y e}\right)^{*}$ the inverse of the transpose of the matrix S34:

$$
\xi_{g}=\operatorname{ArcCos}\left(\frac{18+27 \gamma^{2}+5 \gamma^{4}}{2\left(3+\gamma^{2}\right) \mathrm{A}}\right)
$$

The obliquity rotation axis written in the hexagonal basis is

$$
\boldsymbol{\omega}_{g}=\frac{3-\gamma^{2}}{2 \sqrt{2}\left(3+\gamma^{2}\right)}[\overline{2}, \overline{2}, 1]
$$

The rotation matrix required to compensate the tilt of the plane $\mathbf{g}_{0}=(012)_{p}$ can thus be calculated, but its analytical expression depending on the packing ratio is too large to fit the page width. In the case of ideal $\gamma$ ratio, the obliquity rotation angle is $\xi_{g}=\operatorname{ArcCos}\left(\frac{113}{17} \sqrt{\frac{5}{221}}\right) \approx 1.11^{\circ}$ and the rotation axis in the hexagonal basis is $\boldsymbol{\omega}_{g}=\frac{1}{17 \sqrt{2}}[\overline{2}, \overline{2}, 1]$.

The obliquity-corrected distortion matrix is noted $\mathbf{D}_{\text {hex }}^{g r \rightarrow y e}$. The analytical expressions of the nine components $\mathbf{D}_{i j}$ of $\mathbf{D}_{\text {hex }}^{g r \rightarrow y e}$ depending on the stacking ratio calculated with Mathematica are: 


$$
\begin{aligned}
& \mathbf{D}_{11}=\frac{2\left(-1+\sqrt{\frac{3+7 \gamma^{2}}{3+\gamma^{2}}}\right)+\gamma^{2}\left(2+\sqrt{\frac{3+7 \gamma^{2}}{3+\gamma^{2}}}\right)}{2\left(4+\gamma^{2}\right)} \\
& \mathbf{D}_{12}=\frac{-6-3 \gamma^{2}-\gamma^{4}+2 \mathrm{~A}}{\left(4+\gamma^{2}\right) \mathrm{A}} \\
& \mathbf{D}_{13}=\frac{11 \gamma^{2}+5 \gamma^{4}-6(1+\mathrm{A})}{\left(4+\gamma^{2}\right) \mathrm{A}} \\
& \mathbf{D}_{21}=\frac{-3+2 \gamma^{2}+\gamma^{4}-\mathrm{A}}{12+7 \gamma^{2}+\gamma^{4}} \\
& \mathbf{D}_{22}=\frac{2\left(3+6 \gamma^{2}+\gamma^{4}+\mathrm{A}\right)}{\left(4+\gamma^{2}\right) \mathrm{A}} \\
& \mathbf{D}_{23}=\frac{2\left(3+5 \gamma^{2}+2 \gamma^{4}-3 \mathrm{~A}\right)}{\left(4+\gamma^{2}\right) \mathrm{A}} \\
& \mathbf{D}_{31}=\frac{3-2 \gamma^{2}-\gamma^{4}+\mathrm{A}}{24+14 \gamma^{2}+2 \gamma^{4}} \\
& \mathbf{D}_{32}=\frac{9+\gamma^{2}-\mathrm{A}}{\left(4+\gamma^{2}\right) \mathrm{A}} \\
& \mathbf{D}_{33}=\frac{3\left(9+7 \gamma^{4}+7 \mathrm{~A}+3 \gamma^{2}(8+\mathrm{A})\right)}{\left(4+\gamma^{2}\right) A^{2}}
\end{aligned}
$$

with $A=\sqrt{\left(3+\gamma^{2}\right)\left(3+7 \gamma^{2}\right)}$

In the case of ideal hard-sphere packing the distortion matrix takes the value:

$$
\begin{aligned}
& \mathbf{D}_{\text {hex }}^{g r \rightarrow y e}=\left(\begin{array}{ccc}
\frac{1}{4}+\frac{7 \sqrt{\frac{13}{85}}}{4} & \frac{3}{10}-\frac{19}{2 \sqrt{1105}} & -\frac{9}{10}+\frac{53}{2 \sqrt{1105}} \\
\frac{1}{4}-\frac{3 \sqrt{\frac{13}{85}}}{4} & \frac{3}{10}+\frac{47}{2 \sqrt{1105}} & -\frac{9}{10}+\frac{11 \sqrt{\frac{5}{221}}}{2} \\
-\frac{1}{8}+\frac{3 \sqrt{\frac{13}{85}}}{8} & -\frac{3}{20}+\frac{21}{4 \sqrt{1105}} & \frac{9}{20}+\frac{81}{4 \sqrt{1105}}
\end{array}\right) \\
& \approx\left(\begin{array}{ccc}
0.9344 & 0.0142 & -0.1028 \\
-0.0433 & 1.0069 & -0.0727 \\
0.0217 & 0.0079 & 1.0592
\end{array}\right)
\end{aligned}
$$

It can checked that $\mathbf{D}_{\text {hex }}^{g r \rightarrow y e}$ leaves invariant the direction $\boldsymbol{u}_{0}=[\overline{2}, \overline{2}, 1]_{g r}$ and leaves untilted the plane (012) gr

The orientation of the unconventional twin is given by the misorientation matrix between the hexagonal bases. It is $\mathbf{T}_{\text {hex }}^{g r \rightarrow y e}=\mathbf{D}_{\text {hex }}^{g r \rightarrow y e}\left(\mathbf{C}_{\text {hex }}^{y e \rightarrow g r}\right)^{-1}$ 


$$
\begin{aligned}
& \mathbf{T}_{\text {hex }}^{g r \rightarrow y e} \\
& =\left(\begin{array}{ccc}
\frac{-6-3 \gamma^{2}-\gamma^{4}+2 A}{\left(4+\gamma^{2}\right) A} & \frac{2\left(3-\gamma^{2}\left(2+\gamma^{2}\right)+A\right)}{\left(4+\gamma^{2}\right) A} & -\frac{2 \gamma^{2}\left(7+3 \gamma^{2}+A\right)}{\left(4+\gamma^{2}\right) A} \\
\frac{2\left(3+6 \gamma^{2}+\gamma^{4}+A\right)}{\left(4+\gamma^{2}\right) A} & \frac{-6-11 \gamma^{2}-3 \gamma^{4}+2 A}{\left(4+\gamma^{2}\right) A} & -\frac{2 \gamma^{2}\left(-1+\gamma^{2}+A\right)}{\left(4+\gamma^{2}\right) A} \\
\frac{9+\gamma^{2}-\mathrm{A}}{\left(4+\gamma^{2}\right) A} & \frac{18}{3+7 \gamma^{2}-5 A} & \frac{-12+\gamma^{2}(-8+A)}{\left(4+\gamma^{2}\right) A}
\end{array}\right)
\end{aligned}
$$

with $A=\sqrt{\left(3+\gamma^{2}\right)\left(3+7 \gamma^{2}\right)}$

This orientation matrix is fully equivalent by internal symmetry to the matrix $\mathbf{T}_{h e x}^{g r \rightarrow y e} \mathbf{2}_{100}$, which is a rotation around the axis $\boldsymbol{u}_{0}=[\overline{2}, \overline{2}, 1]_{\text {gr }}$ and of angle

$$
\theta_{D}=\operatorname{ArcCos}\left(\frac{-3-2 \gamma^{2}}{\mathrm{~A}}\right)
$$

In the case of hard-sphere packing, this angle is $\theta_{D}=\operatorname{ArcCos}\left(-5 \sqrt{\frac{5}{221}}\right) \approx 138.77^{\circ}$.

S4. New conventional twin generated by the composition of the unconventional twins derived from the $\left(58^{\circ}, a+2 b\right)$ and $\left(86^{\circ}, b\right)$ twin prototypes.

The calculations were performed with Mathematica (see Supplementary Program Part E).

From the previous calculations and from the EBSD results, the crystallographic link between the parent, green and yellow twins can be summarized as follows:

1. The green twin results from an unconventional $(212)_{p} \rightarrow(012)_{g r}$ twinning of the parent crystal. The correspondence, distortion and orientation matrices associated with this twin mode are $\mathbf{C}_{\text {hex }}^{g r \rightarrow p}, \mathbf{D}_{\text {hex }}^{p \rightarrow g r}$ and $\mathbf{T}_{\text {hex }}^{p \rightarrow g r}$ given by equations S17, S23, and S25.

2. The yellow twins are linked to the green twins by an unconventional $(012)_{g r} \rightarrow(212)_{y e}$ twinning relationship that is an obliquity-corrected form of extension twinning. The correspondence, distortion and orientation matrices associated with this twin mode are $\mathbf{C}_{\text {hex }}^{y e \rightarrow g r}, \mathbf{D}_{\text {hex }}^{g r \rightarrow y e}$ and $\mathbf{T}_{\text {hex }}^{g r \rightarrow y e}$ given by equations S35, S40, and S42, respectively.

3. The yellow twins formed by the combination of the two unconventional twins, i.e. $\boldsymbol{g}_{\mathbf{0}}=$ $(212)_{p} \rightarrow(012)_{g r}$ followed by $(012)_{g r} \rightarrow(212)_{y e}$, appears as a conventional twin relatively to the parent crystal because the plane $\boldsymbol{g}_{\mathbf{0}}=(212)_{p}$ is restored, i.e. $(212)_{p} \rightarrow$ (212) ye

4. The direction $\boldsymbol{u}_{\mathbf{0}}=[0 \overline{2} 1]_{p}$ is maintained invariant by the three twinning modes, only its indexes are changed into equivalent ones: $[0 \overline{2} 1]_{p} \rightarrow[\overline{2} \overline{2} 1]_{g r} \rightarrow[0 \overline{2} 1]_{y e}$

Now, let us define the crystallographic properties of the conventional (parent $\rightarrow$ yellow) twin. Its correspondence, distortion and orientation matrices are determined by combination of the matrices determined in the previous sections. 


\section{S4.1.1. Composition of the correspondence matrices}

The first correspondence $\mathbf{C}_{\text {hex }}^{g r \rightarrow p}$ is followed by the second correspondence $\mathbf{C}_{\text {hex }}^{y e \rightarrow g r}$. Their composition is simply

$$
\begin{gathered}
\mathbf{C}_{\text {hex }}^{y e \rightarrow p}=\mathbf{C}_{\text {hex }}^{y e \rightarrow g r} . \mathbf{C}_{\text {hex }}^{g r \rightarrow p}=\left(\begin{array}{ccc}
\frac{1}{4} & \frac{5}{8} & \frac{5}{4} \\
1 & -\frac{1}{2} & 1 \\
\frac{1}{4} & \frac{1}{8} & -\frac{3}{4}
\end{array}\right) \\
\text { and }\left(\mathbf{C}_{\text {hex }}^{y e \rightarrow p}\right)^{*}=\left(\begin{array}{ccc}
\frac{1}{4} & 1 & \frac{1}{4} \\
\frac{5}{8} & -\frac{1}{2} & \frac{1}{8} \\
\frac{5}{4} & 1 & -\frac{3}{4}
\end{array}\right)
\end{gathered}
$$

\section{S4.1.2. Composition of the distortion matrices}

The first distortion $\mathbf{D}_{h e x}^{p \rightarrow g r}$ is followed by the second distortion $\mathbf{D}_{\text {hex }}^{g r \rightarrow y e}$. It is necessary to work in the same basis to compose these active matrices. The matrix $\mathbf{D}_{h e x}^{g r \rightarrow y e}$ expressed in the parent hexagonal basis becomes $\mathbf{T}_{\text {hex }}^{p \rightarrow g r} . \mathbf{D}_{\text {hex }}^{g r \rightarrow y e}$. $\left(\mathbf{T}_{\text {hex }}^{p \rightarrow g r}\right)^{-1}$. The composition is thus

$$
\begin{gathered}
\mathbf{D}_{\text {hex }}^{p \rightarrow y e}=\mathbf{T}_{\text {hex }}^{p \rightarrow g r} \cdot \mathbf{D}_{\text {hex }}^{g r \rightarrow y e} \cdot\left(\mathbf{T}_{\text {hex }}^{p \rightarrow g r}\right)^{-1} \cdot \mathbf{D}_{\text {hex }}^{p \rightarrow g r} \\
=\left(\begin{array}{ccc}
\frac{33}{28}-\frac{30}{7\left(3+7 \gamma^{2}\right)} & \frac{5\left(-3+\gamma^{2}\right)}{8\left(3+7 \gamma^{2}\right)} & \frac{5\left(-3+\gamma^{2}\right)}{4\left(3+7 \gamma^{2}\right)} \\
\frac{-3+\gamma^{2}}{3+7 \gamma^{2}} & \frac{3+15 \gamma^{2}}{6+14 \gamma^{2}} & \frac{-3+\gamma^{2}}{3+7 \gamma^{2}} \\
-\frac{1}{4}+\frac{6}{3+7 \gamma^{2}} & -\frac{1}{8}+\frac{3}{3+7 \gamma^{2}} & \frac{3}{4}+\frac{6}{3+7 \gamma^{2}}
\end{array}\right)
\end{gathered}
$$

It is checked that $\operatorname{Det}\left(\mathbf{D}_{\text {hex }}^{p \rightarrow y e}\right)=1$, and that the directions $[0 \overline{2} 1]_{p}$ and $[\overline{1} 01]_{p}$ are invariant by $\mathbf{D}_{\text {hex }}^{p \rightarrow y e}$ whatever the packing ratio. This proves that $\mathbf{D}_{\text {hex }}^{p \rightarrow y e}$ is a simple shear matrix that leaves invariant the plane (212) hex . Consequently, $\mathbf{D}_{\text {hex }}^{p \rightarrow y e}$ is a conventional twinning matrix. The shear vector $\boldsymbol{s}$ is calculated by considering the normalized vector perpendicular to the plane $\boldsymbol{g}=(212)_{p}$ expressed in the orthonormal basis, i.e. $\boldsymbol{n}=\frac{\mathbf{H}_{h e x}^{*} \boldsymbol{g}}{\left\|\mathbf{H}_{\text {hex }}^{*} \boldsymbol{g}\right\|}$. The shear vector is

$$
\boldsymbol{s}_{\text {ortho }}=\left(\mathbf{D}_{\text {ortho }}^{p \rightarrow \text { yellow }}-\mathbf{I}\right) \cdot \mathbf{n}
$$

When expressed in the hexagonal basis it becomes

$$
\boldsymbol{s}_{\text {hex }}=\left(\mathbf{H}_{\text {hex }}\right)^{-\mathbf{1}} \cdot \boldsymbol{s}_{\text {ortho }}=\frac{3-\gamma^{2}}{\gamma \sqrt{9+21 \gamma^{2}}}[\overline{5}, \overline{4}, 7]_{\text {hex }}
$$

In four-index notation this vector is of type $\langle 1,2, \overline{3}, 7\rangle_{\text {hex }}$. The shear amplitude is given by its norm, that can be calculated directly from $\boldsymbol{s}_{\text {ortho }}$. It is 


$$
s=\sqrt{\frac{7}{48}} \frac{\left|3-\gamma^{2}\right|}{\gamma}
$$

In the case of hard-sphere packing ratio, the analytical expression of the distortion matrix takes rational values:

$$
\mathbf{D}_{\text {hex }}^{p \rightarrow y e}=\left(\begin{array}{ccc}
\frac{51}{52} & -\frac{1}{104} & -\frac{1}{52} \\
-\frac{1}{65} & \frac{129}{130} & -\frac{1}{65} \\
\frac{7}{260} & \frac{7}{520} & \frac{267}{260}
\end{array}\right) \approx\left(\begin{array}{ccc}
0.9808 & -0.0096 & -0.01922 \\
-0.0154 & 0.9923 & -0.0154 \\
0.0269 & 0.0135 & 1.0269
\end{array}\right)
$$

The shear value along the direction $[\overline{5}, \overline{4}, 7]_{\text {hex }}$ is $s=\frac{1}{24} \sqrt{\frac{7}{2}} \approx 0.078$.

\section{S4.1.3. Composition of the coordinate transformation matrices}

The first coordinate transformation $\mathbf{T}_{h e x}^{p \rightarrow g r}$ is followed by the second coordinate transformation $\mathbf{T}_{\text {hex }}^{g r \rightarrow y e}$. The composition of these passive matrices is simply

$$
\mathbf{T}_{\text {hex }}^{p \rightarrow y e}=\mathbf{T}_{\text {hex }}^{p \rightarrow g r} . \mathbf{T}_{\text {hex }}^{g r \rightarrow y e}=\left(\begin{array}{ccc}
\frac{-3+3 \gamma^{2}}{3+7 \gamma^{2}} & \frac{5 \gamma^{2}}{3+7 \gamma^{2}} & \frac{10 \gamma^{2}}{3+7 \gamma^{2}} \\
\frac{8 \gamma^{2}}{3+7 \gamma^{2}} & -\frac{3\left(1+\gamma^{2}\right)}{3+7 \gamma^{2}} & \frac{8 \gamma^{2}}{3+7 \gamma^{2}} \\
\frac{6}{3+7 \gamma^{2}} & \frac{3}{3+7 \gamma^{2}} & \frac{3-7 \gamma^{2}}{3+7 \gamma^{2}}
\end{array}\right)
$$

An equivalent orientation matrix is obtained by using the internal symmetry $\mathbf{2}_{210}$; it is a rotation around the $\boldsymbol{u}_{0}=[0 \overline{2} 1]_{p}$ axis and of angle

$$
\theta_{D}=\operatorname{ArcCos}\left(\frac{-6+11 \gamma^{2}}{6+14 \gamma^{2}}\right)
$$

With the ideal hard sphere packing ratio, the disorientation matrix is rational

$$
\mathbf{T}_{\text {hex }}^{p \rightarrow y e} \mathbf{2}_{210}=\left(\begin{array}{ccc}
\frac{11}{13} & -\frac{8}{13} & -\frac{16}{13} \\
\frac{31}{65} & \frac{33}{65} & -\frac{64}{65} \\
\frac{27}{65} & -\frac{9}{65} & \frac{47}{65}
\end{array}\right)
$$

And the rotation angle is $\theta_{D}=\operatorname{ArcCos}\left(\frac{7}{13}\right)=57.42^{\circ}$

As the rotation between the parent and yellow twins $(p \rightarrow y e)$ is around the axis $\boldsymbol{u}_{0}=[0 \overline{2} 1]_{p}$ and as this direction is also left invariant by the rotation associated with the $(p \rightarrow g r)$ twin and by the rotation associated with the ( $g r \rightarrow y e)$ twin, it implies that the rotation angles should be linked by an addition. The rotation angles around the $\boldsymbol{u}_{0}$ axis are given in equations S28, S43 and S51, for the $(p \rightarrow g r),(g r \rightarrow y e)$ and $(y e \rightarrow p)$ twins. Even if not obvious, it is indeed checked that

$$
-\operatorname{ArcCos}\left(\frac{6-\gamma^{2}}{2 \sqrt{\left(3+\gamma^{2}\right)\left(3+7 \gamma^{2}\right)}}\right)+\operatorname{ArcCos}\left(\frac{-3-2 \gamma^{2}}{\sqrt{\left(3+\gamma^{2}\right)\left(3+7 \gamma^{2}\right)}}\right)=\operatorname{ArcCos}\left(\frac{-6+11 \gamma^{2}}{6+14 \gamma^{2}}\right)
$$


and thus also in the particular case of hard-sphere packing:

$$
-\operatorname{ArcCos}\left(\sqrt{\frac{5}{221}}\right)+\operatorname{ArcCos}\left(-5 \sqrt{\frac{5}{221}}\right)=\operatorname{ArcCos}\left(\frac{7}{13}\right)
$$

The disorientation, i.e. the equivalent rotation with the minimum rotation angle in absolute value, is obtained with the internal symmetry $\mathbf{2}_{110}$. The disorientation $\mathbf{T}_{\text {hex }}^{p \rightarrow y e} \mathbf{2}_{110}$ is a rotation around the axis $[\overline{2} 21]_{p}$ and of angle

$$
\theta_{D}=\operatorname{ArcCos}\left(\frac{-6+13 \gamma^{2}}{6+14 \gamma^{2}}\right)
$$

With the ideal hard sphere packing ratio, the disorientation matrix is rational

$$
\mathbf{T}_{\text {hex }}^{p \rightarrow y e} \mathbf{2}_{110}=\left(\begin{array}{ccc}
\frac{8}{13} & \frac{3}{13} & -\frac{16}{13} \\
-\frac{33}{65} & \frac{64}{65} & -\frac{64}{65} \\
\frac{9}{65} & \frac{18}{65} & \frac{47}{65}
\end{array}\right)
$$

and the rotation angle is $\theta_{D}=\operatorname{ArcCos}\left(\frac{43}{65}\right)=48.58^{\circ}$.

As the direction $[\overline{2} 21]_{p} \equiv[241]_{p}$ by internal symmetry, the calculated disorientation $\left(48.5^{\circ},[\overline{2} 21]_{p}\right)$ between the parent crystal and the yellow twin fits exactly that obtained in the EBSD map (Figure1c).

\section{S5. Summary of the calculations}

In order to get a better overview of the crystallographic characteristics of the twinning modes described in the previous sections, a table summarizing the main equations is given below:

\begin{tabular}{|c|c|c|c|c|}
\hline Twin mode & $\begin{array}{c}\text { Correspondence } \\
\text { matrix }\end{array}$ & Distortion matrix & $\begin{array}{c}\text { Misorientation } \\
\text { matrix }\end{array}$ & $\begin{array}{c}\text { Angle of rotation } \\
\text { around the axis } \\
\mathbf{u}_{0}\end{array}$ \\
\hline$(p \rightarrow g r)$ & $\mathrm{S} 17$ & $\mathrm{~S} 23$ & $\mathrm{~S} 25$ & $\mathrm{~S} 28$ \\
\hline$(g r \rightarrow y e)$ & $\mathrm{S} 35$ & $\mathrm{~S} 40$ & $\mathrm{~S} 42$ & $\mathrm{~S} 43$ \\
\hline$(p \rightarrow y e)$ & $\mathrm{S} 44$ & $\mathrm{~S} 45$ & $\mathrm{~S} 50$ & $\mathrm{~S} 51$ \\
\hline
\end{tabular}

Table S2 Summary of the main crystallographic equations related to the three twin modes. The last column gives the rotation angle associated with the misorientation matrix chosen among the equivalent ones such that the rotation axis is $\boldsymbol{u}_{0}=[0 \overline{2} 1]_{p}=[\overline{2} \overline{2} 1]_{g r}=[0 \overline{2} 1]_{y e}$. 